\title{
On the Ability to Inhibit Complex Movements: A Stop-Signal Study of Typewriting
}

\author{
Gordon D. Logan \\ Erindale College, Mississauga, Ontario, Canada
}

\begin{abstract}
Experienced typists were instructed to stop typing when they detected a stop signal (a tone or a change in the display). The main question addressed in four experiments was whether typing responses were organized into words or into units smaller than the word (single letters or letter clusters). If subjects typed whole words after the stop signal, the probability of inhibiting the last letter of a word should be close to zero and should not be affected by word length. In fact, the probability was usually closer to one and was strongly affected by word length (see Experiments 1 and 2), suggesting that the unit is smaller than the word. If subjects typed whole words after the stop signal, they should type fewer letters after the signal the longer the stop-signal delay because fewer letters remain to be typed. For a given stop-signal delay, they should type more letters after the signal the longer the word because more letters remain to be typed. Neither of these predictions was confirmed whether subjects typed single words (see Experiments 1, 2, and 4) or sentences (see Experiment 3), again suggesting that the unit is smaller than the word. The only exception occurred in Experiment 3, where subjects tended to type "the" and the space following it before stopping. Experiment 4 compared the stopping task with stopping the current sequence and beginning a new one and found no difference. These two tasks did differ from a third task in which subjects typed two words in succession.
\end{abstract}

This article reports an investigation of the control of typewriting. Skilled typists typed single words or sentences. When a stop signal (a tone) was presented, they were to stop typing as quickly as they could. With this technique, subjects' ability to stop when the stop signal occurs reflects their ability to control their responses: Responses that can be stopped are clearly controlled, whereas responses that cannot be stopped are clearly beyond control. Thus, the latter, apparently uncontrollable responses may be considered ballistic (see Logan, 1981). The major concern in the pres-

This research was supported by Natural Sciences and Engineering Research Council of Canada Grant U0053.

I am grateful to Mohini Kukde and Linda Rechia for testing the subjects and helping with the project. I am especially grateful to the secretaries of Erindale College, who served so willingly as subjects in the experiments and provided many insights in discussions about typing. I would like to thank Margaret Jean Intons-Peterson and three anonymous reviewers for very thorough criticism that improved the article substantially.

Requests for reprints should be sent to Gordon D. Logan, Department of Psychology, Erindale College, University of Toronto, Mississauga, Ontario, Canada, L5L 1 C6. ent experiments was whether the units that are processed ballistically in typing are words or single letters.

Two dependent variables were used to identify the ballistically processed units: the probability of inhibiting the last keystroke of a word and the number of letters typed after the stop signal. The probability of inhibiting the last keystroke should be close to zero regardless of where the stop signal occurs in the word if skilled typists type words ballistically (i.e., they should always type all the letters in a word). By contrast, if typists type letters or letter clusters ballistically, then the probability of inhibiting the last letter should not be zero, but rather, should depend on the time elapsing between the onset of the stop signal and the keystroke for the last letter (i.e., the time available in which to stop).

The second dependent variable is the number of characters typed after the stop signal, which I call the stopping span (see Logan, 1983). It reflects the number of keystrokes typists are committed to typing at any moment. If skilled typists type words ballistically, then they should always complete the 
word they are currently typing. That is, more letters should be typed when the stop signal occurs near the beginning of a word, and fewer letters should be typed when the stop signal occurs near the end of a word. Further, for a given stop signal, more postcue letters should be typed for longer words than for shorter words because more letters remain to be typed in longer words. By contrast, if single letters or letter clusters are the units of ballistic processing in typing, then the stopping span should be relatively uniform over the different times at which the stop signal occurs, relative to the onset of the word, and should not vary with word length.

These predictions were tested in three experiments that involved single words and in a fourth experiment that involved sentences.

\section{Experiment 1}

The first experiment examined a time-contingent version of the stop-signal paradigm. Typists were to type single words, and on $20 \%$ of the trials, a stop signal occurred at a prespecified delay after the onset of the word. Thus, the presentation of the stop signal was contingent on the onset of the word and was independent of the response the subject was preparing or executing. The delays $(500,650$, 800 , and $950 \mathrm{msec}$ ) were selected so that some stop signals occurred before the subject began typing and others occurred while the subject was typing. The former permit an evaluation of the subject's degree of commitment to a typing response before it begins. Studies of choice reaction time suggest that subjects can inhibit single key-press responses up to the point of execution (Logan, 1981); it would be interesting to see if that were the case with the more complex responses in copy typing.

The early delays also yield an index of the extent to which subjects are committed to typing whole words before execution begins. If they are committed, they should type out whole words after a stop signal, even when the stop signal occurs before they begin typing. The longer delays, when the stop signal occurs after subjects have begun typing, permit a determination of the extent to which subjects are committed to typing whole words after execution begins.

\section{Method}

Subjects. The subjects were 12 secretaries from Erindale College who volunteered their services for a small monetary compensation. On the speed test described below, they typed an average of 60.7 words per minute (ranging from 47 to 79 ) with an average error rate of $4.2 \%$ (ranging from $1.9 \%$ to $7.6 \%$ ). None of these subjects had served in any other experiments on typing.

Apparatus and stimuli. The words were written in capital letters, and each letter was formed by illuminating about 20 points in a $5 \times 7$ matrix on a cathode ray tube (CRT; Tektronics Model 604 equipped with P31 phosphor) under the control of a PDP 11/03 laboratory computer. One dot was illuminated every 78 microsec. The brightness of words of different length was kept the same by "painting" extra letters at a nonexistent position on the screen before refreshing the display, so the total number of letters painted on the screen was always seven.

Viewed at a distance of $120 \mathrm{~cm}$, each letter subtended $.19^{\circ} \times .29^{\circ}$ of visual angle. Three-, five-, and seven-letter words were centered on the screen, and they subtended horizontal visual angles of $.76^{\circ}, 1.29^{\circ}$, and $1.86^{\circ}$, respectively. Because the position of the subject's head was not restrained (e.g., by a headrest), these figures are only approximate. The words were exposed for $1,000 \mathrm{msec}$, preceded by a fixation point that was exposed for 500 msec and was extinguished immediately before the word appeared. The intertrial interval was $2,000 \mathrm{msec}$ and began as soon as the word was extinguished.

The stop signal was a $500-\mathrm{msec}, 900 \mathrm{~Hz}$ tone, played through a speaker behind the CRT at a comfortable listening level. It was presented at one of four delays $(500$, 650,800 , and $950 \mathrm{msec}$ ) following the onset of the word.

The keyboard on which subjects typed the words was a Digital Equipment Corporation LA36 DECwriter II, which also served as a terminal for the computer. A special purpose assembly language routine registered keystrokes without echoing them back to the terminal. Thus, subjects had no visual record of what they typed. Timing of keystrokes was accurate to $1 \mathrm{msec}$.

The copy to be typed was a set of 600 words; 200 words from each of three word lengths (three, five, and seven letters) were drawn from the Kucera and Francis (1967) frequency norms. The average frequencies were $171.9(S E=19.1), 179.3(S E=6.0)$, and 168.4 $(S E=$ 9.7) for three-, five-, and seven-letter words, respectively. There were no significant differences in frequency between words of different lengths. The words within each length condition were balanced for hand repetition and alternation in the keystrokes they required because several investigators have shown that successive keystrokes from different hands (alternations) are typed faster than successive keystrokes from the same hand (repetitions; see Ostry, 1977; Sternberg, Monsell, Knoll, \& Wright, 1978). The number of repetitions at each serial position (left to right) was 95 and 105 for three-letter words, 95 , 105,106 , and 96 for five-letter words, and 107, 112, 106, 101,111 , and 127 for seven-letter words.

Procedure. Each subject practiced typing text on the DECwriter and was given a speed test before beginning the experimental trials. The practice consisted of copying a passage from a high school typing text for $2 \mathrm{~min}$. (Jonsson, 1970). The speed test involved typing a different 
passage from the same book for another $2 \mathrm{~min}$. The total number of words each subject typed during the test was divided by two to estimate her typing speed, and the proportion of words in which errors occurred was calculated to estimate her accuracy.

After practice and the speed test, each subject completed 600 experimental trials in which the words were presented one by one on the CRT. There were 200 words of each length (three, five, and seven letters). The stop signal occurred on $20 \%$ of the trials (i.e., 120 times) at one of the four delays $(500,650,800$, and $950 \mathrm{msec})$. The stop signal occurred equally often at each stop-signal delay and each word length (i.e., 10 times at each combination). The order of stop-signal versus no-stop-signal trials, stop-signal delays, and word lengths varied randomly over the experiment. A different random order was prepared for each subject.

The instructions described the sequence of events on trials with no stop signal, and subjects were told to type the words as quickly and as accurately as possible. Then they were told that a tone would sound occasionally and that they should stop typing whenever they heard the tone. They were told not to wait for the stop signal but to type as naturally as possible. Thus, the instructions emphasized the typing task over the stopping task.

The experimental trials were given in five blocks of 120 , and subjects were allowed brief rests between blocks. We also made sure that they remembered the instructions, and we answered any questions that they raised.

\section{Results and Discussion}

Probability of inhibiting keystrokes. The probability of inhibiting the last letter is presented in Figure 1 (part A) as a function of word length and stop-signal delay. The data are means across subjects, and each mean is based on 120 observations.

If subjects tend to type words as wholes, the probability of inhibiting the last letter should be uniformly low. Instead, it was rel- atively high and dropped appreciably only at the longer stop-signal delays with three-letter words when the whole word was nearly typed by the time the stop signal occurred. Clearly, subjects did not often type words as wholes. Analysis of variance (ANOVA) on the probabilities of inhibiting the last letter revealed significant main effects of stop-signal delay, $F(3,33)=75.76, p<.01, M S_{\mathrm{e}}=.008$, and word length, $F(2,22)=69.77, p<.01$, $M S_{\mathrm{e}}=.036$, and a significant interaction between them, $F(6,66)=13.83, p<.01$, $M S_{\mathrm{e}}=.014$.

The pattern in the inhibition data can be accounted for by differences in latency to particular keystrokes: The probability of inhibition was higher for keystrokes with longer latencies. For example, responses to the last letter in a word had longer latencies than did responses to the first letter $(1,613 \mathrm{msec}$ vs. $721 \mathrm{msec}$ ) and were easier to inhibit (the probability of inhibition was .807 vs. .066 ). Further, word length, which increased latency to the last letter but had no effect on latency to the first letter, increased the probability of inhibiting the last letter but had no effect on the probability of inhibiting the first letter. Last letter latencies were $1,214,1,598$, and $2,028 \mathrm{msec}$ for three-, five-, and sevenletter words, respectively; first letter latencies were 736,710 , and $716 \mathrm{msec}$. The corresponding probabilities of inhibition were $.550, .896$, and .975 for the last letter of three-, five-, and seven-letter words, respectively, and $.061, .069$, and .069 for the first letter.

This pattern suggests that the critical vari-

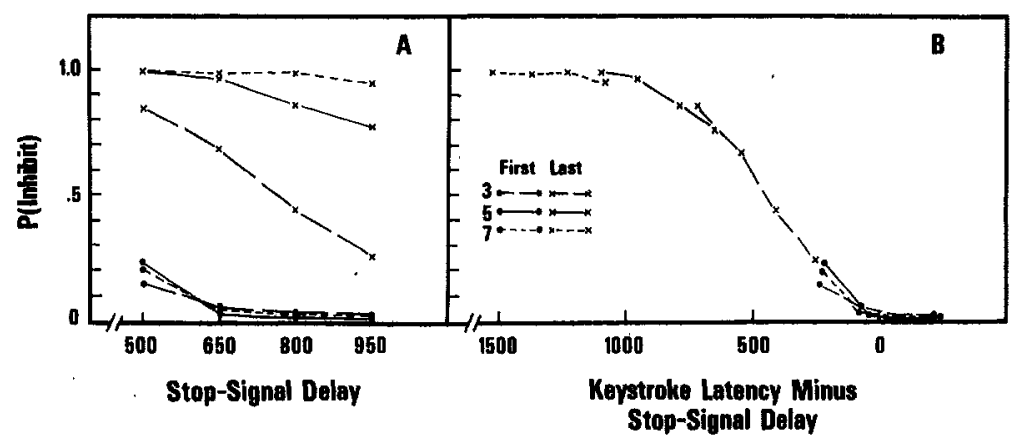

Figure 1. Probability of inhibiting-P(Inhibit) -the first and last letters of a word as a function of stopsignal delay (A) and as a function of the latency to the keystroke minus stop-signal delay (B) in Experiment 1. (Word length, 3, 5, and 7 letters, is the parameter). 
able determining the probability of inhibiting a particular keystroke might be the time elapsing between the onset of the stop signal and the (expected) occurrence of the keystroke, which represents the time available to detect the stop signal before the response occurs. Probabilities of inhibiting the last letters are plotted in Figure 1 (part B) as a function of this variable (i.e., latency to the last keystroke minus stop-signal delay). At each word length, the functions for the last letters align themselves nicely, suggesting that the delay between the onset of the stop signal and the (expected) occurrence of the keystroke was an important determinant of the probability of inhibition; the significant effect of word length and the significant interaction between word length and stop-signal delay are absorbed by this variable. The probabilities of inhibiting the first letter of the word are also plotted in Figure 1 (parts A and B) for comparison. Note that they align themselves with the functions for the last letter in Figure 1 (part B).

$A$ similar pattern of results is found when variants of the stop-signal method are applied to choice reaction time (Logan, 1981) and eye movements (Lisberger, Fuchs, King, \& Evinger, 1975). In those studies, the probability of inhibiting single key-press responses and single eye movements depended only on the interval between the onset of the stop signal and the (expected) occurrence of the response.

Figure 1 (part B) suggests that the context in which the keystroke is executed has little effect on subjects' ability to inhibit it. Points representing stop signals that occurred before typing began (i.e., the two leftmost points for each word length) were on the same function as points representing stop signals that occurred after typing was under way (i.e., the two rightmost points for each word length). This suggests that the response to the stop signal was the same whether subjects were preparing or executing responses. Also, the probability of inhibiting keystrokes in words of different length fell on the same function, suggesting that the ability to inhibit does not depend on the number of keystrokes being prepared.

Stopping span. The mean number of keystrokes typed after the stop signal occurred
Table 1

Means and Standard Deviations of the Number of Letters Typed After the Stop Signal in Experiment 1

\begin{tabular}{crrrr}
\hline & \multicolumn{5}{c}{ Stop-signal delay (in msec) } \\
\cline { 2 - 5 } $\begin{array}{c}\text { Word length } \\
\text { (in letters) }\end{array}$ & 500 & 650 & 800 & 950 \\
\hline Three & & & & \\
$M$ & 1.35 & 1.60 & 1.47 & 1.28 \\
$S D$ & .74 & .81 & .64 & .57 \\
Five & & & & \\
$M$ & 1.14 & 1.74 & 1.83 & 1.88 \\
$S D$ & .80 & .96 & .98 & .87 \\
Seven & & & & \\
$M$ & 1.18 & 1.57 & 1.78 & 2.03 \\
$S D$ & .80 & .92 & .83 & 1.03 \\
\hline
\end{tabular}

Note. Standard deviations are the means of the withinsubject standard deviations.

is presented in Table 1 as a function of stopsignal delay and word length. Each mean in the table is based on 120 observations.

These data suggest that subjects rarely typed whole words after the stop signal occurred. If they had typed whole words, the mean span should have decreased with stopsignal delay because fewer letters remain to be typed at the longer delays, and spans should increase with word length because at each stop-signal delay, more letters remain to be typed with longer words. However, the data in Table 1 generally disconfirm these predictions. For five- and seven-letter words, the span increased with stop-signal delay instead of decreasing, and it did not increase with word length $(M s=1.65$ and 1.64 for five- and seven-letter words, respectively). For three-letter words, span increased from the 500-msec delay to the 650-msec delay but decreased at the longer delays when subjects had nearly completed the word before the signal occurred. This resulted in a lower mean span for three-letter words (1.43 letters) than for five- and seven-letter words.

These effects resulted in significant main effects of stop-signal delay, $F(3,33)=6.92$, $p<.01, M S_{\mathrm{e}}=.283$, and word length, $F(2$, $22)=6.14, p<.01, M S_{\mathrm{e}}=.124$, and a significant interaction between them, $F(6,66)=$ $6.98, p<.01, M S_{\mathrm{e}}=.086$, in an ANOVA on the mean spans.

The increase in span with stop-signal delay suggests that the span may get longer as sub- 
jects progress through the response sequence. However, much of the increase can be accounted for by assuming that span reflects the latency of the (internal) response to the stop signal and that the latency of the response to the stop signal is constant over stop-signal delay. (Logan, 1981, found that this was true for stop signals in a choice reaction time task.) If it were true for typing, we would expect subjects to type fewer letters when the stop signal occurred before they began typing than when the stop signal occurred while they were typing. Table 1 reveals that most of the increase in span with stop-signal delay occurred at the 500- and 650-msec delays before subjects began typing. The increase in span for seven-letter words from the 800- to 950-msec delay was significant, $p<.05$ by Fisher's least significant difference (LSD) test (see Kirk, 1968), and cannot be accounted for by assuming constant latency to the stop signal (because subjects were already typing when the stop signal occurred). Hence the span appears to increase as subjects progress through the response sequence, although this effect did not replicate in Experiments 2, 3, and 4.

\section{Experiment 2}

Using a time-contingent paradigm, Experiment 1 showed that skilled typists can stop typing anywhere within a word as soon as they detect the stop signal. Experiment 2 was conducted to determine whether event-contingent stop signals (i.e., signals presented when particular keystrokes occurred) would have the same effects. This question is important because there is too much variability in typing for time-contingent stop signals to be of much use in studies that use long strings of characters or text. Event-contingent stop signals overcome the problem of typing variability; signals can be presented within one keystroke of a desired location (i.e., contingent on the previous keystroke) no matter how variable typing has been up to that point. However, event-contingent stop signals may introduce problems of their own. In particular, subjects may detect the contingency and alter their typing or their stopping span in response to it. The major purpose of Experiment 2 was to find out whether or not this was the case.
In Experiment 2, stop signals occurred on $20 \%$ of the trials at one of four positions in the response sequence. The routine that accepted responses from the keyboard was modified to present a stop signal when the first, second, third, or fourth keystroke was registered. Naturally, this excluded three-letter words, so the number of trials was reduced from 600 to 400 . In all other respects, the second experiment was a replication of the first.

\section{Method}

Subjects. Twelve secretaries from Erindale College volunteered their services for a small remuneration. Because of the limited number of skilled typists available, 5 of the 12 subjects had served in Experiment 1; only 7 of the 12 had not served in a typing experiment before. Overall, their mean speed on the typing test was 68.4 words per minute (ranging from 53 to 89), and their mean error rate was $3.4 \%$ (ranging from $0 \%$ to $6.8 \%$ ). The mean speed was faster than the one observed in Experiment 1 largely due to the fact that the new subjects ( $M=70.7$ words per minute) were faster than the repeaters ( $M=65.2$ words per minute).

Apparatus and stimuli. The display system, the keyboard, and the control system were the same as in Experiment 1 except that the routine that accepted responses from the keyboard was rewritten to present a stop signal when a prespecified number of keystrokes had been registered (i.e., immediately after the $n$th keystroke). The copy to be typed was the five- and sevenletter words from the first experiment making a total of 400 words.

Procedure. The procedure was largely the same as in Experiment 1: Subjects practiced typing text, performed a speed test, and then performed the experiment itself. This time, there were 400 experimental trials, 200 of each word length (five and seven letters). The stop signal occurred on $20 \%$ of the trials ( 80 times) at one of four delays (after 1, 2, 3, or 4 keystrokes had been registered). The stop signal occurred equally often at each delay and at each word length (10 times), and the order of trials was randomized. Each subject received a different random order. The 400 trials were divided into five blocks of 80 .

\section{Results and Discussion}

Probability of inhibiting keystrokes. The probability of inhibiting the last letter is presented in Figure 2 (left panel) as a function of word length and stop-signal delay. Each point in the figure is based on 120 observations.

Once again, subjects rarely typed whole words. The probability of inhibiting the last letter decreased with stop-signal delay, and the decrease was greater for five-letter words 
than for seven-letter words; the main effects of delay, $F(3,33)=73.88, p<.01, M S_{\mathrm{e}}=$ .017 , and word length, $F(1,11)=103.62$, $p<.01, M S_{\mathrm{e}}=.029$, and the interaction between them, $F(3,33)=43.88, p<.01, M S_{\mathrm{e}}=$ .017 , were significant.

The meaning of these effects is made clearer in Figure 2 (right panel) where the probability of inhibiting the last letter is plotted as a function of the number of letters remaining to be typed when the stop signal occurred (which is ordinally equivalent to keystroke latency minus stop-signal delay, as plotted in Experiment 1). As in Experiment 1 , the data from the different word lengths align themselves nicely, suggesting that the critical variable is the amount of time or keystrokes remaining when the stop signal occurs. This is similar to the single-response data discussed earlier (Lisberger et al., 1975; Logan, 1981). The alignment of data also suggests, as it did in Experiment 1, that typing responses may be stopped at any point in the response sequence; there was no tendency to type whole words.

Stopping span. The span data also show no evidence of a tendency to type whole words (see Table 2).

Based on 120 observations, the means suggest that the span was not substantially affected by word length or stop-signal delay except when signals occurred on the fourth letter of five-letter words. In this situation, only one letter was left to be typed. Clearly, there was no tendency to type whole words.

The truncated span in the fourth position of five-letter words resulted in a significant

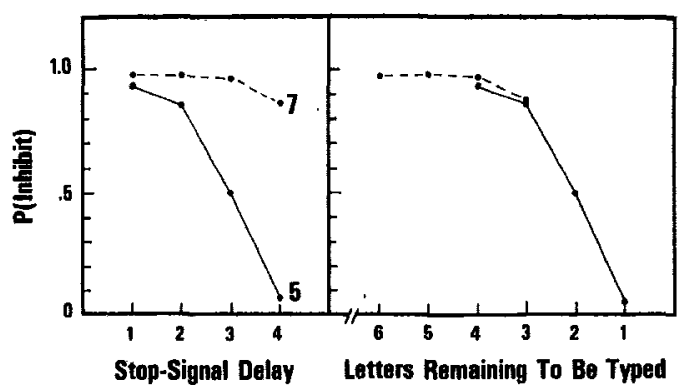

Figure 2. Probability of inhibiting-P(Inhibit)-the last letter in the word as a function of stop signal delay (left panel) and as a function of the number of letters remaining to be typed (right panel) in Experiment 2 . (Word length, 5 and 7 letters, is the parameter in both panels).
Table 2

Means and Standard Deviations of the Number of Letters Typed After Stop. Signal

in Experiment 2

\begin{tabular}{crrrr} 
& \multicolumn{5}{c}{$\begin{array}{c}\text { Stop-signal delay (at positions of } \\
\text { keystroke within words) }\end{array}$} \\
\cline { 2 - 5 } $\begin{array}{c}\text { Word length } \\
\text { (in letters) }\end{array}$ & 1st & 2nd & 3rd & 4th \\
\hline Five & & & & \\
$M$ & 1.38 & 1.50 & 1.44 & .94 \\
$S D$ & .74 & .66 & .44 & .13 \\
Seven & & & & \\
$M$ & 1.43 & 1.61 & 1.41 & 1.54 \\
$S D$ & .87 & .82 & .71 & .71 \\
\hline
\end{tabular}

Note. Standard deviations are average within-subjects standard deviations.

main effect of word length, $F(1,11)=8.61$, $p<.05, M S_{\mathrm{e}}=.092$, a marginally significant main effect of stop-signal delay, $F(3,33)=$ $2.85, p<.06, M S_{\mathrm{e}}=.138$, and a significant interaction between them, $F(3,33)=14.57$, $p<.01, M S_{\mathrm{e}}=.033$. Fisher's LSD test showed that the only point to differ significantly from the others was the fourth position in five-letter words $(p<.01)$.

\section{Experiment 3}

The third experiment was conducted to measure the stopping span in a longer response sequence and to determine whether subjects type whole words as ballistic units in a sentence context. The five- and sevenletter words from Experiments 1 and 2 were used to construct 300 sentences of the form, "the [noun] [verb]ed the [noun]," which were presented one at a time on the CRT for subjects to type. Stop signals occurred on one third of the trials, at one of 20 positions in the sentence. Twelve of these positions were within words (i.e., the first four letters of the first noun, the verb, and the second noun). Spans from these positions could be compared with each other to evaluate the effects of position in the sentence or with spans from Experiment 2 (which also used the first four positions within words) to evaluate the effects of the sentence context. The other eight positions tested were between words (i.e., the last letter and the space following the first four words of the sentence).

Note that the stop signal occurred on a 
higher proportion of trials in this experiment than it did in the previous ones. This may be important because Logan (1981) found that subjects tended to respond more slowly when the stop signal occurred more frequently. However, the present experiment displayed five words each trial, and the proportion of words that occurred with a stop signal $(7 \%)$ was much lower than it was in the previous experiments. Thus, the proportion of signals was higher or lower than before, depending on whether trials or words were the units of analysis. This makes it difficult to predict how performance here should differ from performance in the previous experiments.

There was one further difference between this experiment and the previous ones. In this experiment, the display was extinguished at the onset of the stop signal, whereas in the previous experiments, the duration of the display was independent of the stop signal. It was necessary to extinguish the display in order to speed up the point-plotting routine so that it could display the longer character strings without flicker. ${ }^{1}$

\section{Method}

Subjects. Twelve Erindale secretaries volunteered their services for a small remuneration. Three of them had served in the first experiment, five had served in the second, and four had served in the first and the second. Their mean typing speed was 75.5 words per minute, ranging from 61.5 to 91 . Their mean error rate was $3.13 \%$, ranging from $0 \%$ to $8.73 \%$.

Apparatus and stimuli. The computer, the display system, and the keyboard were the same as in the previous experiments except for two changes. First, in order to present sentences in a single line on the screen, the letters were made smaller, but the viewing distance was reduced so that the visual angle was about the same; from a viewing distance of $70 \mathrm{~cm}$, each letter subtended about $.18^{\circ}$ of visual angle horizontally and $.28^{\circ}$ vertically, compared with $.19^{\circ}$ horizontally and $.29^{\circ}$ vertically in Experiments 1 and 2 . All sentences began at the same position, 14 characters to the left of the center of the screen, and were roughly centered. The average sentence subtended $6.77^{\circ}$ horizontally.

The second change was that the display was extinguished when the stop signal came on. This was necessary to increase the speed at which points were plotted to prevent flicker in the displays. In the previous display routine, the tone was turned on during an interrupt routine that responded to input from the keyboard, and it turned off $500 \mathrm{msec}$ later during the display routine. In order to turn off the tone at the right time, the clock was read after each point was plotted. This step was eliminated in the revised routine (because the display terminated when the tone went on), increasing the speed of plotting from one point per 78 microsec to one point per 38 microsec. This was enough to prevent visible flicker.

There was no fixation point in this experiment; the sentence appeared at the beginning of the trial with no warning. ${ }^{2}$ The sentence was exposed for $5 \mathrm{sec}$, and then it was extinguished, beginning a 2.5 sec intertrial interval. Subjects who type 50 or more words per minute would be able to complete every sentence in the $7.5 \mathrm{sec}$ allowed for typing.

The copy to be typed was a set of 300 sentences of the form, "the [noun] [verb]ed the [noun]," made from the five- and seven-letter words from Experiments 1 and 2. The nouns were either 5 or 7 letters long, but the verbs were $5,6,7$, or 8 letters long because letters sometimes had to be added to make sense. The sentences averaged 28.3 characters in length (including spaces), ranging from 25 to 32 .

Procedure. The procedure was the same as in the previous experiments. Each subject practiced typing, did the speed test, and then performed the experimental trials. There were 300 trials, and the stop signal occurred on $33 \%$ of them ( 100 times) at one of 20 positions in the sentence (five times at each position). Twelve of the positions were within words (the first, second, third, and fourth letters of the first noun, the verb, and the second noun), and eight of the positions were between words (the last letter and the following space from the first four words of the sentence). The order of sentences, stopsignal versus no-stop-signal trials, and stop-signal delays was random. A separate random order was constructed for each subject.

Subjects were instructed as before (i.e., to type quickly

\footnotetext{
${ }^{1}$ Terminating the display with the onset of the stop signal raised the possiblity that the spans would be limited because there was no longer sufficient perceptual data available to drive the typing processes. This possibility was ruled out by demonstrating that subjects were always able to type out more than they did when the stop signal occurred. Two subjects were run under two conditions with the display system from Experiment 3. In one condition, they stopped on signal, and in the other condition, they typed out all they could remember when the signal occurred and the display terminated. Both subjects always typed out all the remaining characters when asked to type all they could remember, demonstrating clearly that they could type more than they did in the stop condition. Data from the no-stop-signal trials suggested that typing was quantitatively similar in the two conditions: Initial latencies were slower in the type-everything condition than in the stop condition (1,093 msec vs. $747 \mathrm{msec})$, but subjects typed at a slightly faster rate in the type-everything condition than in the stop condition (128 msec per character vs. $153 \mathrm{msec}$ per character).

${ }^{2}$ However, the timing was regular and the sentence always began in the same position on the screen, so subjects could prepare themselves adequately in advance if they chose to do so. Apparently, preparation was no problem: The mean initial latency was $629 \mathrm{msec}$, compared with $721 \mathrm{msec}$ in Experiment 1 and $663 \mathrm{msec}$ in Experiment 3. Further, preparation should affect initial latency much more than it affects the stopping span,
} which is the primary focus of the study. 
and accurately and to consider the typing task more important than the stopping task). They were told not to type periods or to type carriage returns at the ends of sentences. The 300 trials were divided into five blocks of 60 , and brief rests were allowed between blocks.

\section{Results and Discussion}

The mean stopping spans are displayed in Table 3 and Table 4 . Table 3 contains withinword spans from the first four letters of the first noun, the verb, and the second noun. Table 4 contains between-word spans from the last letter and the space following the first "the," the first noun, the verb, and the second "the." The 20 means in the two tables were analyzed in one 20-level, one-way ANOVA, which revealed significant differences between conditions, $F(19,209)=5.65, p<.01$, $M S_{\mathrm{e}}=.358$. Several comparisons were noteworthy.

First, the contrast between spans within words and spans between words addresses the extent to which words are typed ballistically in a sentence context. If words tend to be typed as wholes, spans should be larger within words than between words (assuming it is harder to stop within units than between units). The mean within-word span (2.14 letters) was not different from the mean between-words span (2.18 letters), $F(1,209)<$ 1. However, the within-word spans from the last two positions in the last noun were exceptionally small, and the between-words

Table 3

Means and Standard Deviations of the Number of Characters Typed After Stop Signal in Experiment 3

\begin{tabular}{lrrrr}
\hline & \multicolumn{4}{c}{ Positions within words } \\
\cline { 2 - 5 } $\begin{array}{l}\text { Position in } \\
\text { the sentence }\end{array}$ & 1st & 2nd & 3rd & 4th \\
\hline $\begin{array}{l}\text { First noun } \\
M\end{array}$ & & & & \\
$S D$ & 2.15 & 2.65 & 2.25 & 2.20 \\
Verb & .63 & .63 & 1.08 & .98 \\
$M$ & & & & \\
$S D$ & 2.28 & 2.50 & 2.20 & 2.67 \\
Second noun & .88 & 1.01 & .97 & 1.23 \\
$M$ & & & & \\
$S D$ & 2.10 & 2.15 & 1.45 & 1.13 \\
& 1.08 & 1.18 & 1.14 & .93 \\
\hline
\end{tabular}

Note. Standard deviations are average within-subjects standard deviations; position within words refers to the keystroke on which the stop signal was contingent (i.e., the 1st, 2nd, 3rd, and 4th keystroke in each word).
Table 4

Means and Standard Deviations of the Number of Characters Typed After the Stop Signal Between Words in Experiment 3

\begin{tabular}{lrrrr}
\hline \multirow{2}{*}{$\begin{array}{c}\text { Position } \\
\text { between } \\
\text { words }\end{array}$} & \multicolumn{4}{c}{ Sentence position } \\
\cline { 2 - 5 } & 1 & 2 & 3 & 4 \\
\hline Last letter & & & & \\
$M$ & 1.90 & 1.68 & 2.72 & 2.30 \\
$S D$ & .80 & .80 & 1.00 & .92 \\
Space & & & & \\
$M$ & 1.95 & 2.42 & 2.60 & 1.88 \\
$S D$ & .82 & .96 & 1.01 & .84 \\
\hline
\end{tabular}

Note. Standard deviations are average within-subjects standard deviations; Sentence Position 1 refers to signals presented with the last letter and the space following the first "the," Position 2 refers to signals following the first noun, Position 3 refers to signals following the verb and preceding the second "the," and Position 4 refers to signals following the second "the."

spans before, the second "the" were exceptionally large. (The reasons for this are discussed later.) When these exceptional spans were excluded, the remaining within-word spans were larger than the remaining between-word spans (2.36 letters vs. 2.02 letters, respectively), but the difference was not significant by Sheffe's test (Kirk, 1968). Thus, there was no statistical evidence that words tend to be typed ballistically in a sentence context. However, the difference was not small enough to provide much confidence in the null hypothesis, so it may be best to reserve judgment.

Analysis of spans within words also provides evidence on the tendency to type words ballistically: If words are typed ballistically, span should decrease the later in the word the signal occurs. There was no tendency for the span to decrease in the first noun and the verb; the only positions to differ significantly from the rest were the last two positions in the last noun ( $p<.01$ by Fisher's LSD test) where the word was nearly completed when the signal occurred (the mean length of the last noun was 5.9 letters). Thus, there was no evidence that words were typed ballistically. The conclusion that the unit of ballistic processing is smaller than the word appears to generalize to words typed in a sentence context.

Spans between words provide some evidence for ballistic units larger than the letter: 
Spans following the verb, preceding "the," were significantly longer than were spans from the other between-word positions (2.66 letters vs. 2.02 letters, respectively, $p<.01$ by Sheffe's test). Spans before "the" tended to include the word and the space following it; subjects often typed five letters when the stop signal occurred just as the last letter of the verb was being typed (i.e., [space], $t, h$, $\mathrm{e}$, [space]) and four letters when the stop signal occurred just as the space after the verb was being typed (i.e., $t, h$, e, [space]). This can be seen in Figure 3, which displays both the span distributions for signals before "the" and the average distributions from the other between-words positions. Note that both distributions for signals before "the" extend to the space following "the" even though the signals were presented with different keystrokes (i.e., the last letter of the verb vs. the space between the verb and "the"). This is perhaps the clearest evidence of a unit larger than one letter that tends to be typed as a whole.

The longer spans before "the" cannot be attributed to differences in the latencies of the keystrokes that comprise the different spans. Although "the" was typed faster than the other words in the sentences, the difference was not enough to account for the differences in span. Summing the interkeystroke intervals from the stop signal to the end of the span indicated that $364 \mathrm{msec}$ were required to type the 2.7-letter span after the last letter of the word before "the," and 322 msec were required to type the 2.6-letter span after the space before "the." By contrast, only $309 \mathrm{msec}$ were required to type the 2.0-letter span after the last letter of all the other words, and $285 \mathrm{msec}$ were required to type the 1.8letter span after the other spaces. The larger spans before "the" took about $46 \mathrm{msec}$ longer to type than did the shorter spans from the other positions.

"The" may be typed as a unit because it is the most frequent word in written English (Kucera \& Francis, 1967), so skilled typists will have typed it more often than any other word. Also, "the" was the most frequent word in the experiment, occurring twice in each sentence, and this may have induced a special strategy whereby "the" was typed ballistically. Thus, the conclusion that "the" is typed ballistically may be limited in generality; more research is necessary to determine whether this is the case.

Spans from the fourth letter of the verb provide some additional evidence for ballistic units larger than the letter. These were the longest within-word spans, possibly because subjects tended to type out familiar suffixes ("-es" and "-ed") before stopping. Of the 300 verbs in the study, 160 ended with "-es" or "-ed," Spans from the fourth letter of these verbs were longer than spans from the fourth letter of the other verbs (3.10 letters vs. 2.23

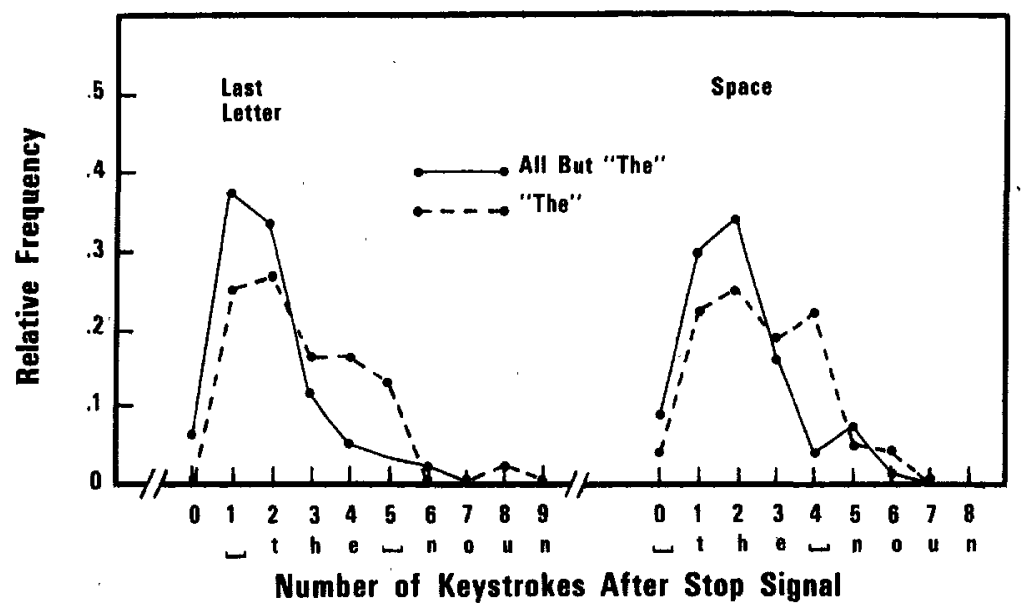

Figure 3. Span distributions before "the" compared with distributions from all other positions between words in Experiment 3. ("Last letter" and "space" refer to the keystroke on which the stop signal occurred; the lettering below the figure indicates the position of "the" relative to the distribution. Both "the" distributions extend to the following space.) 
letters, respectively, $p<.01$ by Fisher's LSD test); suggesting that the familiar suffix was typed ballistically. This comparison must be viewed with caution, however, because the verbs may differ in other ways besides having a familiar suffix appended. Further, the number of observations in the two classes varied randomly between subjects. Indeed, one subject had five verbs that ended in "-es" or "-ed" and no verbs that did not end in "-es" or "-ed" when the fourth position was tested. (Her data were not included in the analysis.) Nevertheless, the elevated spans before familiar suffixes do suggest ballistic units larger than the letter and are worth investigating more systematically.

Averaged across sentence position, the mean span in this experiment was 2.2 characters. This was somewhat larger than the mean spans observed in Experiments 1 and 2 (1.6 and 1.4 characters, respectively) perhaps because of the sentence context. One possibility is that subjects were simply faster in the present experiment so that even if the latency of the response to the stop signal was the same as in the previous experiments, subjects would type more characters before they were able to stop. This possibility was tested by comparing estimates of the latency of the response to the stop signal across experiments (i.e., mean span multiplied by the rate at which characters are typed). In fact, the estimated latencies were longer in Experiment 3 (304 msec vs. 281 and $245 \mathrm{msec}$ in Experiments 1 and 2, respectively), suggesting that there was more to the increased span than an increase in typing speed.

Another possibility is that span increases when subjects type continuous text. This might reflect a shift in the relative importance of the typing task and the stopping task such that subjects are more reluctant to stop when typing sentences than when typing single words. The present data provide no support for the idea that span increases as typists progress through the text.

\section{Experiment 4}

The fourth experiment used a change in the display as a stop signal. Subjects typed single words, and on $25 \%$ of the trials, the word changed into another word at a prespecified delay (i.e., the word change was time contingent). Subjects performed three different tasks when the word changed: In the stop task, they stopped typing when the word changed; in the change task, they stopped typing the first word and began typing the second word; and in the type-both task, they finished typing the first word and then typed the second word.

Contrasts between the three tasks provide insight into the stop-signal procedure: The number of letters typed after the word change in the type-both task provides a quantitative prediction of the size of the stopping span on the hypothesis that typists type whole words ballistically, which can be compared with spans in the stop task and in the change task. The contrast between the number of letters typed after the word change in the change task and in the stop task indicates whether subjects stop differently when they must reprogram the motor system (vs. stopping completely). The contrast between the latency to type the first letter of the second word in the change task and in the type-both task indicates whether the internal components of typing stop when the external components do: We shall see that subjects type fewer letters after the change in the change task than they do in the type-both task, so they are able to stop the overt finger movements in midword. However, they could do so in two different ways: by decoupling the internal and external components and allowing the internal components to run on to completion or by stopping the internal components as well as the external components. If the internal components run on to completion, it should not be possible to begin another overt response until the internal response is finished, in which case latency to type the first letter of the second word should be the same in the change task and in the type-both task. Alternatively, if both internal and external components stop in midword, it should be possible to start another overt response relatively quickly, in which case latency to type the first letter of the second word should be substantially faster in the change task than in the type-both task.

\section{Method}

Subjects. Twelve secretaries and graduate students from Erindale College served in three sessions for a small monetary compensation. One subject was discarded be- 
cause she always typed both words completely in all three tasks. When questioned, she said she thought she was supposed to type both words each session. The mean typing speed for the other 11 subjects was 55.9 words per minute, ranging from 42 to 69 . Their mean error rate was $6 \%$, ranging from $1.6 \%$ to $12.3 \%$. Three subjects had served in the previous experiments, but at least 11 months elapsed since they were last tested.

Apparatus and stimuli. The words to be typed were the five- and seven-letter words from the previous experiments. On each trial, a fixation point was exposed for $500 \mathrm{msec}$. It was followed by a word that was usually exposed for $1 \mathrm{sec}$. On $25 \%$ of the trials, however, the word was extinguished and was replaced by another word at one of four delays $(500,650,800$, and $950 \mathrm{msec})$ after its onset. The second word appeared in the same position as did the first word and remained on for $1 \mathrm{sec}$. A 2-sec intertrial interval began as soon as the last word for the current trial was extinguished. Note that there was no auditory signal when the display changed; subjects had to rely on vision to detect the change. In all other respects, the apparatus and stimuli were the same as in Experiments 1 and 2.

The materials used in the speed test were different from those used in Experiments 1-3. In Experiment 4, the speed test consisted of the first two paragraphs of Miller's (1956) magical number seven paper. It is probably more difficult to read than the texts used in the previous experiments and may have resulted in slower typing speeds. Two of the three subjects who performed in the previous experiments typed the Miller text within one or two words per minute of their speeds on the previous tests; the third subject was slower on the Miller text by about 10 words per minute.

Procedure. Each subject served in three sessions and performed a different task each session. Order of tasks and sessions was balanced between subjects with two subjects receiving each of the six possible orders of the three tasks. Each session involved 320 trials. On 240 trials only one word was presented. Half of these were five-letter words, and half were seven-letter words. On 80 trials, the word changed into another word, thus the probability of change was .25 . Half of the time, the first word was five letters. long, and half of the time it was seven letters long. Half of the time, the second word was the same length as the first and half of the time it was different. This manipulation produced four different conditions, each represented by 20 trials. The word changed at four different delays $(500,650,800$, and 950 msec, as in Experiment 1 , yielding five trials per delay per condition for each subject. The order of change and no-change trials and the order of conditions and delays within the change trials varied randomly. A different random order was prepared each session for each subject.

The speed test was administered on the first session after subjects had practiced typing text on the DECwriter for $2 \mathrm{~min}$. On the second and third sessions, subjects were given $2 \mathrm{~min}$. of text 'typing as a warm up.

Each session began with the warm up, which was followed by the instructions for the task for that session. In each task, subjects were told to type the words as quickly and accurately as possible. They were told to do different things when the word changed: In the stop task, they were told to stop typing as quickly as possible, as in the previous experiments; in the change task, they were told to stop typing as quickly as possible and to begin typing the second word as quickly as possible; in the type-both task, they were told to complete the first word as quickly as possible and then type the second word as quickly as possible.

\section{Results and Discussion}

No-change trials. The contrasts between the spans in the stop and change tasks and between the latencies to the first letter of the second word in the change and type-both tasks assume that performance is equivalent in trials in which the word does not change (i.e., the single-word trials). This assumption was tested by comparing the latency to the first letter of the first word (initial latency) and the time taken to type the first word (the duration of the typing response) in the three tasks. The mean initial latencies in the three tasks appear in Table 5, and the mean durations appear in Table 6. Each mean is based on 1,320 observations. Initial latency to the first word did not vary significantly between tasks, $F(2,20)=3.03, p<.10, M S_{\mathrm{e}}=$ $1,371.90$, and the duration of the first word did not vary either, $F(2,20)=1.27, p>.10$, $M S_{\mathrm{e}}=7,584.49$. Duration of the first word did increase significantly with word length, however, $F(1,10)=298.65, p<.01, M S_{\mathrm{e}}=$ 6,338.99. The lack of an effect of tasks in the initial latency and duration data indicates that subjects were performing equivalently in the three tasks when only one word appeared. Thus, the span data and the initial latencies to the second word can be compared, as if against a common baseline.

Typing after the word change. The mean numbers of letters typed after the word changed in the three tasks are presented in Table 7. Each mean is based on 110 observations.

The mean spans were about the same in

Table 5

Mean Latencies to the First Keystroke of the First and Second Words in Experiment 4

\begin{tabular}{cccccccc}
\hline & \multicolumn{3}{c}{$\begin{array}{c}\text { First-word } \\
\text { task }\end{array}$} & & \multicolumn{2}{c}{$\begin{array}{c}\text { Second-word } \\
\text { task }\end{array}$} \\
\cline { 2 - 4 } $\begin{array}{cccccc}\text { Word length } \\
\text { (in letters) }\end{array}$ & Stop & Change & Both & & Change & Both \\
\hline Five & 687 & 676 & 702 & & 899 & 1181 \\
Seven & 681 & 676 & 700 & & 886 & 1136 \\
\hline
\end{tabular}

Note . Both = type both words. 
Table 6

Mean Durations of the Typing Responses to the First and Second Words in Experiment 4

\begin{tabular}{crrrrrr}
\hline & \multicolumn{3}{c}{$\begin{array}{c}\text { First-word } \\
\text { task }\end{array}$} & & \multicolumn{2}{c}{$\begin{array}{c}\text { Second-word } \\
\text { task }\end{array}$} \\
\cline { 2 - 3 } $\begin{array}{l}\text { Word length } \\
\text { (in letters) }\end{array}$ & Stop & Change & Both & & Change & Both \\
\hline Five & 705 & 713 & 739 & & 726 & 717 \\
Seven & 1045 & 1042 & 1087 & & 1055 & 1100 \\
\hline
\end{tabular}

Note. Both $=$ type both words.

the stop task and in the change task (2.8 letters vs. 3.0, respectively) and were not affected substantially by word length or the delay of the word change. By contrast, the spans in the type-both task were substantially longer (5.0 letters) and were strongly affected by word length and word-change delay.

These observations were supported by ANOVA: When all three tasks were analyzed together, there were significant effects of tasks, $F(2,20)=44.44, p<.01, M S_{\mathrm{e}}=5.567$, word length, $F(1,10)=63.62, p<.01, M S_{\mathrm{e}}=$ 1.370, and word-change delay, $F(3,30)=$ $6.33, p<.01, M S_{\mathrm{e}}=2.089$, and there were significant interactions between tasks and word length, $F(2,20)=60.54, p<.01$, $M S_{\mathrm{e}}=.573$, and tasks and word-change delay, $F(6,60)=22.44, p<.01, M S_{\mathrm{e}}=.633$. However, none of these effects was significant in an analysis of spans from the stop and change tasks by themselves, indicating that the effects in the overall analysis were due to the type-both task.

The qualitative and quantitative differences between the spans in the type-both task on the one hand and the stop and change tasks on the other suggest that the unit of ballistic processing in the stop and change tasks is smaller than the word. Performance in the type-both task represents quantitative predictions of performance in the stop and change tasks on the hypothesis that subjects must type out whole words before stopping; the differences in the size and in the pattern of the span force the rejection of that hypothesis. This conclusion is consistent with the previous experiments, which used a different stop signal.

The finding that spans in the stop task in and the change task did not differ in size or in pattern suggests that subjects stopped typing in equivalent ways in the two tasks. Thus, conclusions about how typing stops in the change task may generalize to the stop task in this experiment and in the previous experiments.

Note that the stopping spans here were nearly twice as long as were the spans observed in Experiment 1, which also used single words and time-contingent stop signals. The signal occurred more often in the present experiment than in Experiment 1 (25\% of the trials vs. $20 \%$ ), and it involved a different sensory modality (vision vs. hearing). I think the difference in span length is due to the difference in the modality of the stop signal. Auditory signals are often more alerting than visual ones and are often responded to faster (Posner, Nissen, \& Klein, 1976). Further, subjects must look at the screen to detect the visual signal but need not look anywhere to detect the tone; any tendency to watch the hands while typing would increase stopping spans with visual signals but not with auditory signals. This is probably more important than is the $5 \%$ difference in the frequency

Table 7

Means and Standard Deviations of the Number of Letters Typed After the Word Changed in Experiment 4

\begin{tabular}{|c|c|c|c|c|}
\hline \multirow{2}{*}{$\begin{array}{l}\text { Task and } \\
\text { word length } \\
\text { (in letters) }\end{array}$} & \multicolumn{4}{|c|}{ Delay of word change (in msec) } \\
\hline & 500 & 650 & 800 & 950 \\
\hline \multicolumn{5}{|l|}{ Stop } \\
\hline \multicolumn{5}{|l|}{ Five } \\
\hline$M$ & 2.43 & 2.88 & 2.94 & 2.60 \\
\hline$S D$ & .71 & 1.02 & .98 & .79 \\
\hline \multicolumn{5}{|l|}{ Seven } \\
\hline$M$ & 2.68 & 3.00 & 2.95 & 3.05 \\
\hline$S D$ & 1.12 & 1.22 & 1.18 & 1.01 \\
\hline \multicolumn{5}{|l|}{ Change } \\
\hline \multicolumn{5}{|l|}{ Five } \\
\hline$M$ & 2.83 & 3.01 & 2.86 & 2.61 \\
\hline$S D$ & .98 & .84 & .80 & .68 \\
\hline \multicolumn{5}{|l|}{ Seven } \\
\hline$M$ & 3.04 & 3.36 & 3.27 & 3.23 \\
\hline$S D$ & 1.05 & 1.15 & 1.09 & 1.18 \\
\hline \multicolumn{5}{|l|}{ Type both } \\
\hline \multicolumn{5}{|l|}{ Five } \\
\hline$M$ & 4.99 & 4.55 & 3.88 & 2.79 \\
\hline$S D$ & .21 & .50 & .71 & 1.04 \\
\hline \multicolumn{5}{|l|}{ Seven } \\
\hline$M$ & 6.85 & 6.35 & 5.64 & 4.70 \\
\hline$S D$ & .40 & .85 & .83 & .85 \\
\hline
\end{tabular}

Note. Standard deviations are average within-subjects standard deviations. 
with which stop signals occurred; Logan (1981) found that a $10 \%$ difference in the frequency with which stop signals occurred had no effect on the latency to detect the signal, though it slowed the primary, choice reaction time task by about 40 msec.

Regardless of the reason for the difference in span length, the pattern of the spans was the same in Experiments 1 and 4; stop-signal delay and word length had the same (null) effect in both experiments. It is this pattern, not the absolute length of the spans, that leads to the conclusion that subjects can inhibit typing in midword.

Typing the second word. The mean initial latency to the second word and the mean duration of the second word in the change and type-both tasks appear in Tables 5 and 6 , respectively. Each mean is based on 110 observations.

The latency to the first letter of the second word was substantially slower in the typeboth task than in the change task $(1,158 \mathrm{msec}$ vs. $892 \mathrm{msec}$ ). Moreover, the length of the first word had a substantial effect in the typeboth task $(1,021 \mathrm{msec}$ when the first word was five letters long; $1,296 \mathrm{msec}$ when it was seven letters long) but not in the change task (855 msec for five-letter first words; $928 \mathrm{msec}$ for seven-letter first words). This likely occurred because subjects had to finish the first word in order to type the second word in the type-both task but could abort the first word in the change task. This difference produced significant effects of task, $F(1,10)=31.21$, $p<.01, M S_{\mathrm{e}}=50,056.05$, length of first word, $F(1,10)=112.37, p<.01, M S_{\mathrm{e}}=$ $5,933.48$, and produced a significant interaction between them, $F(1,10)=45.01, p<$ $.01, M S_{\mathrm{e}}=5,026.08$, in an ANOVA on the second-word initial latencies. These effects were due primarily to the type-both task; when the change-task data were analyzed by themselves, the effect of the length of the first word remained significant, $F(1,10)=11.56$, $p<.01, M S_{\mathrm{e}}=5,027.11$, but it was substantially weaker.

The finding that subjects began typing the second word faster in the change condition than in the type-both condition suggests that subjects were able to inhibit the internal components of the typing response when they inhibited the external components (i.e., overt finger movements). This means that com- mands to the motor system can be overwritten or replaced without having to "run off' the old commands first. ${ }^{3}$

The spans in the change task and in the type-both task took $497 \mathrm{msec}$ and $863 \mathrm{msec}$ to type, respectively. This means that there were $395 \mathrm{msec}$ and $295 \mathrm{msec}$ of "dead time" between the end of the response to the first word and the beginning of the response to the second word in the change task and in the type-both task, respectively. The dead time represents about 2.4 interkeystroke intervals in the change task and 1.7 interkeystroke intervals in the type-both task. One interpretation is that subjects inserted a space (character) between the first and second words in an internal motor program (contrary to instructions but consistent with habit), which they deleted when executing the program. Thus, the dead time should represent about two interkeystroke intervals (i.e., one between the last letter of the first word and the space, and one between the space and the first letter of the second word), which it does. This interpretation suggests that the transition from the first word to the second word was very smooth, even though it was largely unexpected (occurring on $25 \%$ of the trials). The durations of the second word provide some supporting evidence.

The mean durations of the second word in the change and the type-both task appear in Table 6. They were similar to each other and similar to the durations of the first word in all three tasks. Indeed, when the durations of all words in all tasks were compared in one analysis, there was no main effect of tasks, $F(4,40)<1, M S_{\mathrm{e}}=7,510.46$. The effect of word length was highly significant, $F(1,10)=292.72, p<.01, M S_{\mathrm{e}}=11,031.47$, accounting for $99 \%$ of the treatment variance, and the interaction between tasks and word length was also significant, $F(4$,

\footnotetext{
${ }^{3}$ This finding also rules out a degenerate strategy subjects could use to stop quickly, namely, raising their hands from the keyboard and completing the programmed movement sequence without striking the keys. Subjects using this strategy in the change task should not be able to begin the second word until they finish the movements "in the air." Thus, their initial latencies to the second word should be no faster than initial latencies to the second word in the type-both task. The finding that second-word initial latencies were faster in the change task than in the type-both task rules out this possibility.
} 
$40)=3.43, p<.05, M S_{\mathrm{e}}=793.15$, accounting for less than $1 \%$ of the treatment variance. The interaction was due primarily to subjects' tendency to type the second word slower in the type-both condition. However, the difference was relatively small. These data, then, suggest that the transition from the first word to the second word was relatively smooth in that it did not slow the pace of typing adopted for the first word. In particular, the transition in the change task was smooth, indicating that the requirement to abort one complex motor response and to initiate another did not disrupt the flow of typewriting.

\section{General Discussion}

In all four experiments, subjects rarely typed whole words after the stop signal occurred unless they were explicitly instructed to do so. Whether typing single words or sentences, subjects seemed able to inhibit their responses one or two keystrokes after the stop signal. This suggests that the units that are processed ballistically in typing are smaller than the word, perhaps single letters or clusters of two or three. The only important exception was observed in Experiment 3, where subjects tended to type out "the" and the following space as a unit and to complete familiar verb endings ("-es" and "-ed").

Note that the conclusion that the ballistic unit is smaller than the word follows from the effects of word length and stop-signal delay rather than from the absolute length of the spans. If the ballistic unit were the word, stopping span should increase with word length and decrease with stop-signal delay. This pattern failed to materialize in all four experiments, so it seems safe to reject the hypothesis. Subjects seemed able to stop typing in midword.

To what extent might the relatively close control seen in the present typing studies generalize to other tasks, discrete and continuous? In Experiments 1 and 2 (see Figures 1, part B, and 2, right panel), the probability of inhibiting individual keystrokes followed a function very similar to functions describing the probability of inhibiting single button presses in choice reaction time tasks (Logan, 1981 ) and the probability of inhibiting single eye movements in step tracking tasks (Lis- berger et al., 1975). The fact that different keystrokes followed the same function independent of their context suggests that keystrokes can be controlled individually just as discrete responses are controlled individually. The fact that subjects stopped typing very quickly (250-300 msec in Experiments 1-3) suggests that they may be able to stop other, more continuous tasks quickly as well. Vince and Welford (1967) provide evidence that smooth movements of the hands can be stopped quickly. Movements involving more of the body's mass may be more difficult to stop and may take more time to stop for physical reasons (e.g., inertia) rather than psychological reasons.

The present experiments raise very interesting questions about the relation between automaticity and control: Why do skilled typists have such close control over typing if skilled typing is automatic? Typing is nearly a prototypical example of an automatic process. It is a commonplace observation that skilled typists can carry on other activities while they are typing, and Shaffer (1975) has demonstrated formally that typing from visual copy does not suffer interference from a concurrent shadowing task. According to the common view that automatic processes do not suffer interference from concurrent tasks (Logan, 1979; Posner \& Snyder, 1975), this demonstration means that typing is automatic. Yet the skilled typists in the present experiments showed evidence of control over every keystroke.

This apparent contradiction arises because we have learned to think of automaticity and control as opposites (e.g., Shiffrin \& Schneider, 1977). However, they need not be. Automatization may reflect a change in the way a task is controlled, rather than the abdication of control (e.g., Logan, 1979). For example, subjects may shift to a higher level of control as they gain experience on a task (e.g., attending to words rather than individual keystrokes), so that the parameters they were attempting to control at the lower level are adjusted as an inevitable consequence of controlling higher-level parameters (see Pew, 1974). This does not mean the lower-level parameters are any less controlled; the control is simply less direct. Thus, automatization need not involve abdication of control.

To pursue this idea in typewriting, subjects 
may be able to control individual keystrokes directly by engaging a feedback loop that releases the next keystroke when it receives some signal indicating that the current keystroke was under way. In this low-level control system, subjects could stop typing as soon as they hear the stop signal by interrupting the feedback loop. Alternatively, it is possible to control typing by controlling communication between the component processes of typing (e.g., perceptual processes, motor processes, and timing processes). The component processes may be able to function autonomously, without direct control, but the results of their efforts may have no effect on behavior unless they are communicated to the other processes. Thus, control depends on communication between processes (see Logan, 1979). With a high-level control system such as this one, it should be possible to interrupt behavior very quickly, by disrupting communication between the component processes. For example, in typing, the motor system may be set to release keystrokes when it receives a pulse from a timing system (see Shaffer, 1978); if so, subjects could stop typing as soon as they hear the stop signal by preventing the timing pulse from reaching the motor system.

Of course, these control systems are highly speculative and cannot be distinguished by the present data. Nevertheless, they serve to make the point that shifting to a higher level of control in order to perform a task more automatically need not diminish the ability of the control system to adapt to sudden changes, such as stop signals. Indeed, the idea that automatization is an adaptive response, designed to produce the best performance possible given the constraints of the task environment, suggests that new control systems that result from automatization should not afford the subject less control than the more primitive ones. Good control is adaptive and should be preserved whenever possible.

\section{References}

Jonsson, A. S. Timed writings. Ottawa, Ontario: McGrawHill, 1970.

Kirk, R. E. Experimental design: Procedures for the behavioral sciences. Monterey, Calif.: Brooks/Cole, 1968.
Kucera, H., \& Francis, W. N. Computational analysis of present-day American English. Providence, R.I.: Brown University Press, 1967.

Lisberger, S. G., Fuchs, A. F., King, W. M., \& Evinger, L. C. Effect of mean reaction time on saccadic responses to two-step stimuli with horizontal and vertical components. Vision Research, 1975, 15, 10211025.

Logan, G. D. On the use of a concurrent memory load to measure attention and automaticity. Journal of Experimental Psychology: Human Perception and Performance, 1979, 5, 189-207.

Logan, G. D. Attention, automaticity, and the ability to stop a speeded choice response. In J. Long \& A. D. Baddeley (Eds.), Attention and performance (Vol: 9). Hillsdale, N.J.: Erlbaum, 1981.

Logan, G. D. Time, information, and the various spans in typewriting. In W. E. Cooper (Ed.), Cognitive aspects of skilled typewriting. New York: Springer-Verlag, 1983.

Miller, G. A. The magical number seven, plus or minus two: Some limits on our capacity for processing information. Psychological Review, 1956, 63, 81-97.

Ostry, D. J. The organization of typewriting performance (Doctoral dissertation, University of Toronto, 1977). Dissertation Abstracts International, 1978, 39, 3560B-3561B

Pew, R. W. Human perceptual-motor performance. In B. H. Kantowitz (Ed.), Human information processing: Tutorials in performance and cognition. Hillsdale, N.J.: Erlbaum, 1974.

Posner, M. I., Nissen, M. J., \& Klein, R. M. Visual dominance: An information processing account of its origins and significance. Psychological Review, 1976, $83,157-171$.

Posner, M. I., \& Snyder, C. R. R. Attention and cognitive control. In R. L. Solso (Ed.), Information processing and cognition: The Loyola Symposium. Hillsdale, N.J.: Erlbaum, 1975.

Shaffer, L. H. Multiple attention in continuous verbal tasks. In P. M. A. Rabbitt \& S. Dornic (Eds.), Attention and performance (Vol. 5). New York: Academic Press, 1975.

Shaffer, L. H. Timing in the motor programming of typing. Quarterly Journal of Experimental Psychology, 1978, 30, 333-345.

Shiffrin, R. M., \& Schneider, W. Controlled and automatic human information processing: II. Perceptual learning, automatic attending, and a general model. Psychological Review, 1977, 84, 127-190.

Sternberg, S., Monsell, S., Knoll, R. L., \& Wright, C. E. The latency and duration of rapid movement sequences: Comparisons of speech and typewriting. In G. E. Stelmach (Ed.), Information processing in motor control and learning. New York: Academic Press, 1978.

Vince, M. A., \& Welford, A. T. Time taken to change the speed of a response. Nature, 1967, 213, 532-533.

Received November 19, 1981

Revision received April 23, 1982 